\title{
INFLUENCE OF THE ENVIRONMENTAL DARK MATTER ON QSO-CLUSTER ASSOCIATIONS AND ON CLUSTER MASS ESTIMATES
}

\author{
Zuhui FAN ${ }^{1,2,3}$ AND TZIHONG CHIUEH ${ }^{2,3}$ \\ Received 1999 November 30; accepted 2000 February 9
}

\begin{abstract}
The environmental dark matter mass around clusters is investigated by taking into account dark halo-cluster correlations. We find that while the surrounding dark matter significantly increases the lensing amplification factor as compared to that caused by foreground clusters only, the total enhancement factor for background QSOs still remains quite insufficient to explain the observed strong QSOcluster associations. Effects of the environmental matter on the cluster mass estimates through the inversion of weak-lensing distortions of background galaxies are also studied. For relatively small clusters, the existence of the environmental dark matter would lead to an overestimate of the cluster mass, whereas for large clusters, their mass would be underestimated.
\end{abstract}

Subject headings: cosmology: theory — dark matter — galaxies: clusters: general gravitational lensing - large-scale structure of universe

\section{INTRODUCTION}

Gravitational lensing is the most plausible reason for the observed correlation between high- and low-redshift objects (Fugmann 1990; Bartelmann \& Schneider 1992, 1993a, 1993b; Benitez, Martinez-Gonzalez, \& Martin-Mirones 1997; Bartsch, Schneider, \& Bartelmann 1997; RodriguesWilliams \& Hogan 1994; Seitz \& Schneider 1995; Rodrigues-Williams \& Hawkins 1995; Wu \& Han 1995). It has been known for a long time, however, that the lensing amplification caused solely by foreground objects (galaxies or clusters of galaxies) is too weak to explain the observed strong associations. As a result of galaxy-galaxy, galaxycluster, and cluster-cluster correlations, there are excess numbers of galaxies and clusters of galaxies around foreground objects. Quantitative calculations have shown that the contribution to the lensing amplification by the environmental mass from surrounding galaxies and clusters of galaxies is still far less than needed to account for the observational results (e.g., Schneider 1989; Wu \& Fang 1996; Wu et al. 1996).

Compact dark matter halos have been invoked as lenses to explain the appearance of multiple quasars with large angular separations (Narayan \& White 1988; Jaroszynski et al. 1990; Cen et al. 1994; Kochanek 1995; Hawkins 1997; Wambsganss, Cen, \& Ostriker 1998). Weak-lensing effects by large-scale structures of dark matter along lines of sight have also caught attention recently. Observations have revealed significant coherent distortions of galaxies around projected locations of several high-redshift quasars (Fort et al. 1995; Schneider et al. 1998). Since there are no detected clusters in the foreground, the observed shear signals are likely the (biased) cosmic shear caused by inhomogeneous distributions of the dark matter along lines of sight (Schneider et al. 1998). There have been studies to analytically estimate the cosmic shear of background galaxies induced by large-scale structures in the universe (e.g., Jain \&

\footnotetext{
${ }^{1}$ Department of Astronomy and Astrophysics, The University of Chicago, 5640 South Ellis Avenue, Chicago, IL 60637.

2 Department of Physics, National Taiwan University, 1, Roosevelt Road Section 4, Taipei, Taiwan, Republic of China.

${ }^{3}$ Institute of Astronomy \& Astrophysics, Academia Sinica, PO Box 1-87, Nankang, Taipei, Taiwan 115, Republic of China.
}

Seljak 1997; Kaiser 1998; Bartelmann \& Schneider 1999). If galaxies are indeed (biased) tracers of the underlying dark matter, excessive large-scale structures around galaxies due to the galaxy-dark matter correlation should contribute to QSO-galaxy associations. Bartelmann (1995) computed the large-scale-structure-induced QSO-galaxy correlations using the linear power spectrum of dark matter massdensity fluctuations. Recently, similar studies were carried out for nonlinear density perturbations (Dolag \& Bartelmann 1997; Sanz, Martinez-Gonzalez, \& Benitez 1997) by invoking the nonlinear power spectrum of density fluctuations in model calculations (Peacock \& Dodds 1994). At angular scales of several arcminutes, nonlinear effects give rise to significant QSO-galaxy correlations, which are roughly an order of magnitude higher than the linear results.

In this paper, we study the weak-lensing effect of largescale structures of dark matter on QSO-cluster associations. Instead of using the nonlinear power spectrum to directly calculate the QSO-cluster correlation, we adopt the method of Wu et al. (1996). We consider dark matter to be in the form of dark halos of different size. Because of the dark halo-cluster correlation, there should be excessive dark matter halos around foreground clusters. By approximating the density profile of dark halos as a singular isothermal sphere, we can calculate the corresponding dark mass around clusters, and furthermore the lensing amplification and the enhancement factor for background QSOs. The advantage of this approach is that, as noted above, the surrounding dark mass can be estimated directly. Not only can we study the effect of dark matter on the QSO-cluster correlation, but we can also estimate the influence of the environmental dark matter on the estimate of cluster mass calculated through weak-lensing distortions of background galaxies.

There are numerical simulations to scrutinize this projection effect (e.g., Cen 1997; Reblinsky \& Bartelmann 1999). In particular, Wu et al. (1997) numerically studied the QSOcluster correlation caused by large-scale structures of dark matter, and found only a minor number enhancement of QSOs around foreground clusters. However, these simulation results could be compromised by limited numerical 
resolutions. Our analytical approach does not suffer this resolution problem. In addition, we can incorporate different cosmological models relatively easily and analyze the impact of various cosmological parameters on weak-lensing effects induced by large-scale structure, keeping in mind the qualifications of certain simplified assumptions.

The rest of the paper is organized as follows. Section 2 presents the formulation on which our analyses are based. In $\S 3$ we study particular models. Discussions are given in $\S 4$. Throughout the paper, the Hubble constant is taken to be $H_{0}=50 \mathrm{~km} \mathrm{~s}^{-1} \mathrm{Mpc}^{-1}$.

\section{FORMULATION}

The two-point correlation function between a dark halo with the top-hat smoothing radius, $r_{0}$, and a cluster at redshift $z_{c}$ is represented by $\xi_{d c}\left(r, r_{0}, z_{c}\right)$, where $r$ is the proper distance between the dark halo and the cluster. Since the correlation length is much shorter than the cosmological distance to the cluster, we ignore the redshift difference between the dark halo and the cluster (cf. Peebles 1980). It then follows that

$$
r \approx\left[\frac{a^{2}\left(x_{d}-x_{c}\right)^{2}}{1+\left(1-\Omega_{0}\right) a_{0}^{2} x_{c}^{2} /\left(c / H_{0}\right)^{2}}+\eta^{2}\right]^{1 / 2},
$$

where $a$ is the scale factor at $z_{c}$ (the present scale factor $\left.a_{0}=1\right), x_{d}$ and $x_{c}$ are the coordinate distances of the dark halo and the cluster, respectively, and $\eta$ is the separation between the dark halo and the cluster in the cluster plane. By the same approximation, the volume element with $\eta$ in the range $(\eta, \eta+d \eta)$ can be written as

$$
d V=2 \pi \eta d \eta \frac{a d x_{d}}{\left[1+\left(1-\Omega_{0}\right) a_{0}^{2} x_{c}^{2} /\left(c / H_{0}\right)^{2}\right]^{1 / 2}} .
$$

Within this volume, the excess number of dark halos with the top-hat smoothing scale $r_{0}$ is then given by

$$
\begin{aligned}
d N\left(\eta, r_{0}\right)= & (2 \pi \eta d \eta)\left[n\left(r_{0}\right) d r_{0}\right]\left(1+z_{c}\right)^{3} \\
& \times \int_{-\infty}^{\infty} \xi_{d c}\left[\left(\eta^{2}+l^{2}\right)^{1 / 2}, r_{0}, z_{c}\right] d l,
\end{aligned}
$$

where $n\left(r_{0}\right) d r_{0}$ is the comoving number density of dark halos, $\left[n\left(r_{0}\right) d r_{0}\right]\left(1+z_{c}\right)^{3}$ is the proper number density of dark halos at $z_{c}$, and $l \equiv a\left(x_{d}-x_{c}\right) /[1+(1$ $\left.\left.-\Omega_{0}\right) a_{0}^{2} x_{c}^{2} /\left(c / H_{0}\right)^{2}\right]^{1 / 2}$. The integration range is taken to be $(-\infty, \infty)$ because of the sharply peaked behavior of the correlation function (cf. Peebles 1980).

Let $m\left(\eta, \eta_{0}, r_{0}\right)$ be the mass contribution from a dark halo with a smoothing radius $r_{0}$ at $\eta$ to the projected area within $\eta_{0}$ around the cluster. The total mass contribution to this area $\pi \eta_{0}^{2}$ around the cluster due to dark halos with various sizes and at various locations is

$$
\begin{aligned}
M_{d}\left(\eta_{0}\right)= & \int_{0}^{\infty} 2 \pi \eta d \eta \int_{0}^{\infty} d r_{0} n\left(r_{0}\right)\left(1+z_{c}\right)^{3} m\left(\eta, \eta_{0}, r_{0}\right) \\
& \times \int_{-\infty}^{\infty} \xi_{d c}\left[\left(\eta^{2}+l^{2}\right)^{1 / 2}, r_{0}, z_{c}\right] d l .
\end{aligned}
$$

To compute $m\left(\eta, \eta_{0}, r_{0}\right)$, we assume a singular isothermal sphere for the density profile of dark halos. Then the surface mass density of a dark halo is given by

$$
\Sigma\left(\eta^{\prime}\right)=\frac{\sigma_{v}^{2}}{2 G} \eta^{\prime-1}
$$

where $\sigma_{v}$ is the velocity dispersion. To avoid an infinite mass, a cutoff radius of $\eta_{f}$ is introduced, which is related to the mass $M$ of a dark halo by

$$
\eta_{f}=\frac{G M}{\pi \sigma_{v}^{2}} .
$$

The mass of a dark halo with a top-hat smoothing radius $r_{0}$ is $M=(4 \pi / 3) \rho_{0} r_{0}^{3}$, where $\rho_{0}$ is the current mass density of the universe, and thus

$$
\eta_{f}=\frac{H_{0}^{2} \Omega_{0} r_{0}^{3}}{2 \pi \sigma_{v}^{2}},
$$

where $\Omega_{0}$ is the density parameter of the universe, and the relation $\rho_{0}=3 H_{0}^{2} \Omega_{0} /(8 \pi G)$ has been used. For various parameter regimes, the function $m\left(\eta, \eta_{0}, r_{0}\right)$ is now given by

$$
m\left(\eta, \eta_{0}, r_{0}\right)=\frac{\pi \sigma_{v}^{2}}{G} \eta_{f}, \quad \text { for } \eta \leq \eta_{0}, \quad \eta_{f}\left(r_{0}\right) \leq\left(\eta_{0}-\eta\right),
$$

$$
\begin{gathered}
m\left(\eta, \eta_{0}, r_{0}\right)=\frac{\sigma_{v}^{2}}{G} \eta_{f} \tilde{\theta}+\frac{\sigma_{v}^{2}}{G} \\
\quad \times \int_{\tilde{\theta}}^{\pi} d \theta\left[\eta \cos \theta+\left(\eta_{0}^{2}-\eta^{2} \sin ^{2} \theta\right)^{1 / 2}\right], \\
\text { for } \eta \leq \eta_{0}, \quad\left(\eta_{0}-\eta\right)<\eta_{f}\left(r_{0}\right) \leq\left(\eta_{0}+\eta\right), \\
m\left(\eta, \eta_{0}, r_{0}\right)=\frac{\sigma_{v}^{2}}{G} \int_{0}^{\pi} d \theta\left(\eta_{0}^{2}-\eta^{2} \sin ^{2} \theta\right)^{1 / 2}, \\
\text { for } \eta \leq \eta_{0}, \quad \eta_{f}\left(r_{0}\right)>\left(\eta+\eta_{0}\right), \\
m\left(\eta, \eta_{0}, r_{0}\right)=0, \quad \text { for } \eta>\eta_{0}, \quad \eta_{f}\left(r_{0}\right) \leq\left(\eta-\eta_{0}\right), \quad(2.9) \\
m\left(\eta, \eta_{0}, r_{0}\right)=\frac{2 \sigma_{v}^{2}}{G} \int_{\tilde{\theta}}^{\sin -1\left(\eta_{0} / \eta\right)} d \theta\left(\eta_{0}^{2}-\eta^{2} \sin ^{2} \theta\right)^{1 / 2}+\frac{\sigma_{v}^{2}}{G} \\
\times \int_{0}^{\tilde{\theta}} d \theta\left[\eta_{f}-\eta \cos \theta+\left(\eta_{0}^{2}-\eta^{2} \sin ^{2} \theta\right)^{1 / 2}\right], \\
\text { for } \eta>\eta_{0}, \quad\left(\eta-\eta_{0}\right)<\eta_{f}\left(r_{0}\right) \leq\left(\eta+\eta_{0}\right), \quad(2.10)
\end{gathered}
$$

and

$$
\begin{gathered}
m\left(\eta, \eta_{0}, r_{0}\right)=\frac{2 \sigma_{v}^{2}}{G} \int_{0}^{\sin ^{-1}\left(\eta_{0} / \eta\right)} d \theta\left(\eta_{0}^{2}-\eta^{2} \sin ^{2} \theta\right)^{1 / 2}, \\
\text { for } \eta>\eta_{0}, \quad \eta_{f}\left(r_{0}\right)>\left(\eta+\eta_{0}\right),
\end{gathered}
$$

where the angular parameter is

$$
\tilde{\theta}=\cos ^{-1}\left(\frac{\eta_{f}^{2}+\eta^{2}-\eta_{0}^{2}}{2 \eta_{f} \eta}\right) .
$$

The correlation function is modeled as (cf. Bardeen et al. 1986)

$$
\begin{aligned}
\xi_{c d}\left(r, r_{0}, z_{c}\right) & =b_{d}\left(r_{0}, z_{c}\right) b_{c}\left(z_{c}\right) \xi_{\rho}\left(r, z_{c}\right) \\
& =\left[\frac{b_{d}\left(r_{0}, z_{c}\right)}{b_{g}\left(z_{c}\right)}\right] b_{g}\left(z_{c}\right) b_{c}\left(z_{c}\right) \xi_{\rho}\left(r, z_{c}\right) \\
& =\left[\frac{b_{d}\left(r_{0}, z_{c}\right)}{b_{g}\left(z_{c}\right)}\right] \xi_{g c}\left(r, z_{c}\right),
\end{aligned}
$$

where $b_{d}, b_{g}$, and $b_{c}$ are the bias factors for dark halos, galaxies, and clusters, respectively; $\xi_{\rho}$ is the autocorrelation 
function of density fluctuations; and $\xi_{g c}$ is the two-point correlation function between galaxies and clusters. By defining $f\left(r_{0}, z_{c}\right) \equiv\left[b_{d}\left(r_{0}, z_{c}\right) / b_{g}\left(z_{c}\right)\right]$, the total mass contribution is

$$
\begin{aligned}
& M_{d}\left(\eta_{0}\right)=\int_{0}^{\eta_{0}} \eta d \eta\left\{\int_{-\infty}^{\infty} \xi_{g c}\left[\left(\eta^{2}+l^{2}\right)^{1 / 2}, z_{c}\right] d l\right\} \\
& \times \int_{0}^{r_{0}\left(\eta_{0}-\eta\right)} \frac{2 \pi^{2} \sigma_{v}^{2}}{G} \eta_{f} n\left(r_{0}\right)\left(1+z_{c}\right)^{3} f\left(r_{0}, z_{c}\right) d r_{0} \\
& +\int_{0}^{\infty} \eta d \eta\left\{\int_{-\infty}^{\infty} \xi_{g c}\left[\left(\eta^{2}+l^{2}\right)^{1 / 2}, z_{c}\right] d l\right\} \\
& \times \int_{r_{0}\left(\left|\eta_{0}-\eta\right|\right)}^{r_{0}\left(\eta_{0}+\eta\right)} \frac{2 \pi \sigma_{v}^{2}}{G}\left(\eta_{f} \cos ^{-1} \tilde{\theta}-\eta \sin \tilde{\theta}\right) \\
& \times n\left(r_{0}\right)\left(1+z_{c}\right)^{3} f\left(r_{0}, z_{c}\right) d r_{0} \\
& +\int_{0}^{\eta_{0}} \eta d \eta\left\{\int_{-\infty}^{\infty} \xi_{g c}\left[\left(\eta^{2}+l^{2}\right)^{1 / 2}, z_{c}\right] d l\right\} \\
& \times \int_{0}^{\pi} d \theta\left(\eta_{0}^{2}-\eta^{2} \sin ^{2} \theta\right)^{1 / 2} \\
& \times \int_{r_{0}\left(\eta_{0}-\eta\right)}^{\infty} \frac{2 \pi \sigma_{v}^{2}}{G} n\left(r_{0}\right)\left(1+z_{c}\right)^{3} f\left(r_{0}, z_{c}\right) d r_{0} \\
& +\int_{\eta_{0}}^{\infty} \eta d \eta\left\{\int_{-\infty}^{\infty} \xi_{g c}\left[\left(\eta^{2}+l^{2}\right)^{1 / 2}, z_{c}\right] d l\right\} \\
& \times \int_{0}^{\sin ^{-1}\left(\eta_{0} / \eta\right)} d \theta\left(\eta_{0}^{2}-\eta^{2} \sin ^{2} \theta\right)^{1 / 2} \\
& \times \int_{r_{0}\left(\eta-\eta_{0}\right)}^{\infty} \frac{4 \pi \sigma_{v}^{2}}{G} n\left(r_{0}\right)\left(1+z_{c}\right)^{3} f\left(r_{0}, z_{c}\right) d r_{0} \\
& -\int_{0}^{\infty} \eta d \eta\left\{\int_{-\infty}^{\infty} \xi_{g c}\left[\left(\eta^{2}+l^{2}\right)^{1 / 2}, z_{c}\right] d l\right\} \\
& \times \int_{r_{0}\left(\left|\eta_{0}-\eta\right|\right)}^{r_{0}\left(\eta_{0}+\eta_{0}\right)} \frac{2 \pi \sigma_{v}^{2}}{G}\left[\int_{0}^{\tilde{\theta}}\left(\eta_{0}^{2}-\eta^{2} \sin ^{2} \theta\right)^{1 / 2}\right] \\
& \times n\left(r_{0}\right)\left(1+z_{c}\right)^{3} f\left(r_{0}, z_{c}\right) d r_{0},
\end{aligned}
$$

where $r_{0}(x)$ is the smoothing radius satisfying $\eta_{f}\left(r_{0}\right)=x$. Hence, the average surface mass density, $\bar{\Sigma}$, within the area $\pi \eta_{0}^{2}$ due to surrounding dark halos is $\bar{\Sigma}=M_{d}\left(\eta_{0}\right) /\left(\pi \eta_{0}^{2}\right)$.

The Press-Schechter formula (Press \& Schechter 1974; Kochanek 1995) is employed to estimate the number density of dark halos. It is assumed that density fluctuations smoothed over a top-hat comoving scale $r_{0}$ are Gaussian, with an rms linear overdensity (at the present time) of $\Delta\left(r_{0}\right)$. If we denote $\delta_{c}(z)$ as the critical overdensity that can collapse at redshift $z$, the comoving number density of dark halos with top-hat smoothing radius $r_{0}$ is

$$
n\left(r_{0}\right) d r_{0}=\frac{-3 \delta_{c}(z)}{(2 \pi)^{3 / 2} r_{0}^{3} \Delta\left(r_{0}\right) r_{0}} \frac{d \ln \Delta\left(r_{0}\right)}{d \ln r_{0}} \exp \left[-\frac{\delta_{c}^{2}(z)}{2 \Delta^{2}\left(r_{0}\right)}\right],
$$

where the relation between the mass $M$ and $r_{0}$ for the top-hat smoothing, namely, $M\left(r_{0}\right)=(4 \pi / 3) \rho_{0} r_{0}^{3}$, has been used, and $\Delta\left(r_{0}\right)$ is related to the power spectrum, $P(k)=$ $\left|\delta_{k}\right|^{2}$, of density fluctuations by

$$
\Delta^{2}\left(r_{0}\right)=(2 \pi)^{-3} \int_{0}^{\infty} 4 \pi k^{2} d k P(k) W^{2}\left(k r_{0}\right),
$$

where $W(x)$ is the Fourier transform of the top-hat smoothing function,

$$
W(x)=3\left(\frac{\sin x-x \cos x}{x^{3}}\right) .
$$

For the critical overdensity, $\delta_{c}(z)$, we adopt the result from the simple spherical collapse model. For a universe without the cosmological constant, $\delta_{c}(z)$ is related to the density parameter, $\Omega_{0}$, and the redshift, $z$, by

$$
\delta_{c}(z)=\frac{3}{2} \frac{D_{1}\left(\Omega_{0}^{-1}-1\right)}{1-\Omega_{0}}\left\{\left[\frac{\pi \Omega_{0}}{F_{1}(z)}\right]^{2 / 3}+1-\Omega_{0}\right\},
$$

where

$$
D_{1}(x)=1+\frac{3}{x}+\frac{3(1+x)^{1 / 2}}{x^{3 / 2}} \ln \left[(1+x)^{1 / 2}-x^{1 / 2}\right]
$$

and

$$
\begin{aligned}
F_{1}(z)=\{ & \frac{\left(1+\Omega_{0} z\right)^{1 / 2}}{\left(1-\Omega_{0}\right)(1+z)} \\
& \left.-\frac{\Omega_{0}}{2\left(1-\Omega_{0}\right)^{3 / 2}} \cosh ^{-1}\left[\frac{2\left(1-\Omega_{0}\right)}{\Omega_{0}(1+z)}+1\right]\right\} .
\end{aligned}
$$

We assume that a dark halo collapses and virializes to form a singular isothermal sphere with a velocity dispersion $\sigma_{v}$ and a three-dimensional cutoff radius $r_{f}$. The velocity dispersion, $\sigma_{v}$, is given by

$$
\sigma_{v}=H_{0} r_{0} \Omega_{0}^{1 / 3} 2^{-1 / 2} \pi^{1 / 3} F_{1}(z)^{-1 / 3},
$$

and the cutoff radius, $r_{f}$, is, by the relation $M=2 \sigma_{v}^{2} r_{f} / G$,

$$
r_{f}=\frac{G M}{2 \sigma_{v}^{2}}=\frac{H_{0}^{2} \Omega_{0} r_{0}^{3}}{4 \sigma_{v}^{2}} .
$$

Note that $r_{f}$ is related to the two-dimensional cutoff radius $\eta_{f}$ by $\eta_{f}=(2 / \pi) r_{f}$.

In the limit of $\Omega_{0}=1$, one has the following relations: $D_{1}\left(\Omega_{0}^{-1}-1\right) /\left(1-\Omega_{0}\right)=\frac{2}{5}, \quad F_{1}(z)=\frac{2}{3}(1+z)^{-3 / 2}, \quad \delta_{c}(z)=$ $\frac{3}{5}(3 \pi / 2)^{2 / 3}(1+z)$, and $\sigma_{v}=2^{-1 / 2}(3 \pi / 2)^{1 / 3} H_{0} r_{0}(1+z)^{1 / 2}$.

With the prescriptions given above, one can calculate $\bar{\Sigma}$ for specific cosmological models. The lensing amplification factor $\mu$ can be estimated by approximating the dark matter distribution as a uniform sheet with a surface mass density $\bar{\Sigma}$ (Schneider, Ehlers, \& Falco 1992),

$$
\mu=\frac{1}{\left[1-\left(\bar{\Sigma} / \Sigma_{\text {crit }}\right)\right]^{2}},
$$

where the critical surface mass density is given by

$$
\Sigma_{\text {crit }}=\frac{c^{2}}{4 \pi G} \frac{D_{s}}{D_{c s} D_{c}}
$$

where $D_{s}, D_{c}$, and $D_{c s}$ are the angular-diameter distances from the observer to the source, from the observer to the lens (the cluster), and from the lens to the source, respectively. The angular-diameter distance from us to an object 
at redshift $z$ is given by (cf. Schneider et al. 1992)

$$
\begin{aligned}
D(z)= & \frac{c}{H_{0}} \frac{2}{\Omega_{0}^{2}(1+z)^{2}} \\
& \times\left\{\Omega_{0} z-\left(2-\Omega_{0}\right)\left[\left(\Omega_{0} z+1\right)^{1 / 2}-1\right]\right\},
\end{aligned}
$$

and the angular-diameter distance from an object at $z_{1}$ to an object at $z_{2}$ is

$$
\begin{aligned}
D\left(z_{1}, z_{2}\right)= & \frac{c}{H_{0}} \frac{2}{\Omega_{0}^{2}}\left(1+z_{1}\right) \\
& \times\left\{\left[\frac{\Omega_{0} z_{2}-\Omega_{0}+2}{\left(1+z_{2}\right)^{2}}\right]\left[\frac{\left(\Omega_{0} z_{1}+1\right)^{1 / 2}}{\left(1+z_{1}\right)^{2}}\right]\right. \\
& \left.-\left[\frac{\Omega_{0} z_{1}-\Omega_{0}+2}{\left(1+z_{1}\right)^{2}}\right]\left[\frac{\left(\Omega_{0} z_{2}+1\right)^{1 / 2}}{\left(1+z_{2}\right)^{2}}\right]\right\} .
\end{aligned}
$$

The total amplification factor, including the central cluster of galaxies, is

$$
\begin{aligned}
\mu\left(\eta_{0}\right)= & \frac{1}{\left[1-4 \pi\left(\sigma_{c} / c\right)^{2}\left(D_{c s} D_{c} / D_{s}\right)\left(1-\bar{\Sigma} / \Sigma_{\text {crit }}\right)^{-1} / \eta_{0}\right]} \\
& \times \frac{1}{\left[1-\left(\bar{\Sigma} / \Sigma_{\text {crit }}\right)\right]^{2}},
\end{aligned}
$$

where $\eta_{0}$ is the distance to the cluster center at the lens plane, and $\sigma_{c}$ is the velocity dispersion within the cluster.

The enhancement factor, $q$, of QSOs around foreground clusters is (Narayan 1989)

$$
q=\frac{1}{\mu} \frac{N(S / \mu)}{N(S)}
$$

where $N(S)$ is the cumulative surface number density of QSOs with flux larger than $S$, and the $1 / \mu$ factor takes into account the area distortion by the gravitational lensing effect. For a power-law number density distribution, $N(S)$, modeled as

$$
N(S) \propto S^{-\beta},
$$

where the power-law index $\beta$ is assumed to be independent of the QSO redshift, $q$ is simply

$$
q=\mu^{\beta-1} .
$$

\section{RESULTS}

For a cold dark matter universe, the power spectrum of the linear density fluctuation field can be written as (e.g., Efstathiou, Bond, \& White 1992)

$$
P(k)=\frac{B k^{n}}{\left\{1+\left[a k+(b k)^{3 / 2}+(c k)^{2}\right]^{v}\right\}^{2 / v}},
$$

where $a=(6.4 / \Gamma) h^{-1} \mathrm{Mpc}, b=(3.0 / \Gamma) h^{-1} \mathrm{Mpc}, c=(1.7 /$ $\Gamma) h^{-1} \mathrm{Mpc}, v=1.13, \Gamma=\Omega_{0} h, h$ is the Hubble constant in units of $100 \mathrm{~km} \mathrm{~s}^{-1} \mathrm{Mpc}^{-1}, B$ represents the perturbation amplitude, and $n$ is the power index, taken to be $n=1$.

With equations (3.1) and (2.15), one can get $\Delta\left(r_{0}\right)$, the density fluctuation averaged over the smoothing window, $W$. We will use the top-hat smoothing function throughout this paper. For $\Omega_{0}=1$ and $h=0.5, \Delta\left(r_{0}\right)$ can be approximated by (Narayan \& White 1988)

$$
\begin{aligned}
\Delta\left(r_{0}\right) & \approx\left(\frac{2.6}{b_{8}}\right) 6.258 \\
& \times\left[1-0.3909\left(r_{0} h^{-1}\right)^{0.1}+0.4814\left(r_{0} h^{-1}\right)^{0.2}\right]^{-10},
\end{aligned}
$$

where $r_{0}$ is in units of $h^{-1} \mathrm{Mpc}$, and $b_{8}$ is the biasing factor for galaxy clustering, satisfying $b_{8} \Delta\left(8 h^{-1} \mathrm{Mpc}\right)=1$. Equation (3.2) is accurate enough for $0.025 h^{-1} \mathrm{Mpc} \leq r_{0} \leq 20$ $h^{-1}$ Mpc.

For $\Omega_{0}=0.5$ and 0.2 , we find that $\Delta\left(r_{0}\right)$ can be described by the following fitting formulae:

$$
\begin{aligned}
\Delta\left(r_{0}\right) \approx & \Omega_{0}^{2}\left(\frac{5.538}{b_{8}}\right) 6.258\left[1-0.3909\left(r_{0} h^{-1} \Omega_{0}\right)^{0.1}\right. \\
& \left.+0.4814\left(r_{0} h^{-1} \Omega_{0}\right)^{0.195}\right]^{-10},
\end{aligned}
$$

for $\Omega_{0}=0.5$ and $0.025 h^{-1} \mathrm{Mpc}<r_{0}<30 h^{-1} \mathrm{Mpc}$, and

$$
\begin{aligned}
\Delta\left(r_{0}\right) \approx & \Omega_{0}^{2}\left(\frac{17.8283}{b_{8}}\right) 6.258\left[1-0.3909\left(r_{0} h^{-1} \Omega_{0}\right)^{0.1}\right. \\
& \left.+0.4814\left(r_{0} h^{-1} \Omega_{0}\right)^{0.19}\right]^{-10},
\end{aligned}
$$

for $\Omega_{0}=0.2$ and $0.025 h^{-1} \mathrm{Mpc}<r_{0}<50 h^{-1} \mathrm{Mpc}$.

In Figure 1, we plot $\Delta\left(r_{0}\right)$ from equations (3.2)-(3.4) for $b_{8}=1$, along with the results calculated directly from equation (2.15). We see that equations (3.2)-(3.4) give rise to very good approximations for $\Delta\left(r_{0}\right)$.

We use equations (3.2)-(3.4) together with the formulae in the preceding section to calculate the additional dark matter surface density, $\bar{\Sigma}$, around clusters due to clusterdark halo correlations.

We apply the galaxy-cluster correlation function (cf. Peebles 1980)

$$
\xi_{g c}\left(r, z_{c}\right)=\left(1+z_{c}\right)^{-(3+\epsilon)}\left(\frac{r}{r_{g c}}\right)^{-\gamma},
$$

where the correlation length is $r_{g c} \approx 15 h^{-1} \mathrm{Mpc}$, and the correlation power index is $\gamma=1.8$. The factor $\left(1+z_{c}\right)^{-(3+\epsilon)}$

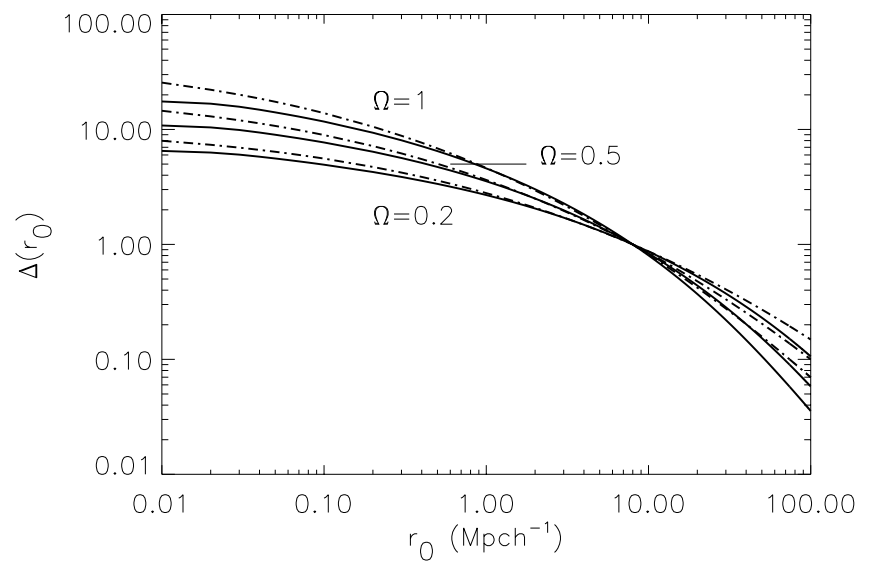

FIG. 1.-Variance of density fluctuations, $\Delta\left(r_{0}\right)$, vs. the top-hat smoothing scale, $r_{0}$. The solid lines show results directly calculated from equation (2.15), and the dash-dotted lines show results from approximate equations (3.2)-(3.4). 
describes the evolution of the correlation function (e.g., Koo $\&$ Szalay 1984; Shepherd et al. 1997). For $\epsilon=0$, the clustering is stable in physical coordinates. With $\epsilon=\gamma-3$, the clustering is fixed in comoving coordinates. For $b_{d}\left(r_{0}, z_{c}\right)$, we adopt the biasing factor given by Bardeen et al. (1986), namely,

$$
b_{d}\left(r_{0}, z_{c}\right) \approx \frac{\delta_{c}\left(z_{c}\right) / \Delta\left(r_{0}\right)}{\Delta\left(r_{0}, z_{c}\right)}+1,
$$

where $\Delta\left(r_{0}, z_{c}\right)$ is the average density perturbation within $r_{0}$ at redshift $z_{c}$. In the linear regime, we have $\Delta\left(r_{0}, z_{c}\right)=$ $\left[\delta_{c}(0) / \delta_{c}\left(z_{c}\right)\right] \Delta\left(r_{0}\right)$, and then

$$
b_{d}\left(r_{0}, z_{c}\right) \approx \frac{\delta_{c}\left(z_{c}\right) / \Delta\left(r_{0}\right)}{\left[\delta_{c}(0) / \delta_{c}\left(z_{c}\right)\right] \Delta\left(r_{0}\right)}+1 .
$$

The galaxy bias factor is approximated by (Bardeen et al. 1986)

$$
b_{g}\left(z_{c}\right) \approx \frac{\langle v\rangle}{\Delta\left(r_{g}, z_{c}\right)}+1,
$$

where $\langle v\rangle$ is the average height of dark matter density perturbation peaks that host galaxies, and $r_{g}$ is the smoothing radius corresponding to galaxies. With $b_{g}(0)=b_{8}=$ $\left[\langle v\rangle / \Delta\left(r_{g}\right)\right]+1$, we have

$$
b_{g}\left(z_{c}\right) \approx \frac{\left(b_{8}-1\right) \Delta\left(r_{g}\right)}{\Delta\left(r_{g}, z_{c}\right)}+1 .
$$

In Figure 2, we show $\left(\bar{\Sigma}(\theta) / \Sigma_{\text {crit }}\right)\left(1+z_{c}\right)^{(3+\epsilon)}$ versus $\theta$, where the redshift of the cluster is $z_{c}=0.2$, the source redshift is $z_{s}=2$, and $b_{8}=1$. One can see that $\bar{\Sigma}(\theta)$ approximately follows the power-law relation of the twodimensional angular correlation function of clusters, but with a power index slightly smaller than 0.8 . Assuming the same clustering evolution (that is, the same $\epsilon$ value) and the same bias factor, $b_{8}$, the dark matter surface density is approximately proportional to $\Omega_{0}$. In order to satisfy observational constraints, e.g., the cluster abundance, however, the normalization factor $B$ of the power spectrum, or equivalently $b_{8}$, is dependent on $\Omega_{0}$. Furthermore, the evolution

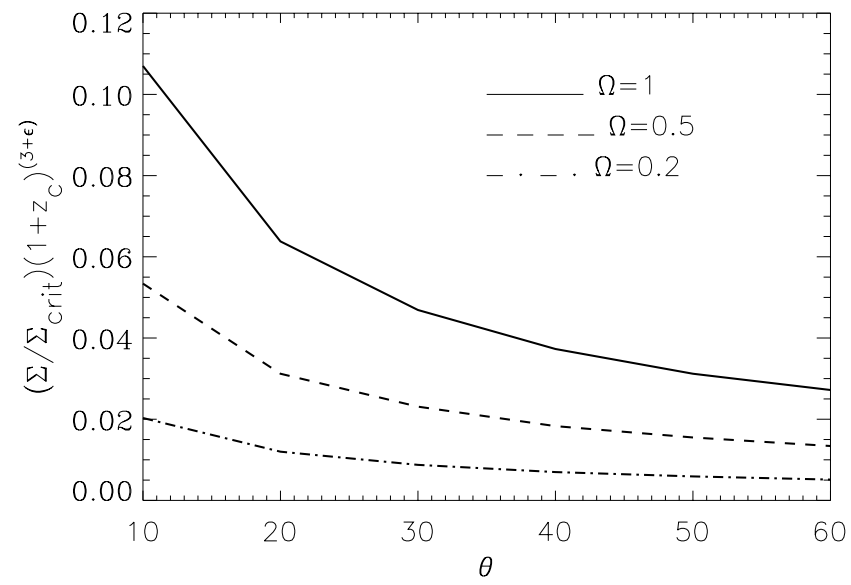

FIG. 2.-Plot of $\left(\bar{\Sigma} / \Sigma_{\text {crit }}\right)\left(1+z_{c}\right)^{(3+\epsilon)}$ vs. $\theta$ (in arcminutes), with source redshift $z_{s}=2$ and cluster redshift $z_{c}=0.2$, and a bias factor $b_{8}=1$ for all three models. of the correlation function also depends on cosmological models. For $\Omega_{0}=1$, the structure clustering should still be dynamically active at present, and a strong evolution for the correlation function is thus expected. For an open universe, the dynamical clustering activity would be relatively quiet at low redshifts, and the correlation function changes mildly from, say, redshift $z \sim 0.2$ to the present epoch. Figure 3 shows $\left(\bar{\Sigma}(\theta) / \Sigma_{\text {crit }}\right)\left(1+z_{c}\right)^{(3+\epsilon)}$ with different values of $b_{8}$ for different models according to the relation $b_{8}^{-1} \Omega_{0}^{0.56}=0.57$ (e.g., White, Efstathiou, \& Frenk 1993). We see that differences in $\bar{\Sigma}(\theta)$ between the three models are reduced to $\bar{\Sigma} \sim$ $\Omega_{0}^{0.4}$. It is expected that the dependence of $\bar{\Sigma}(\theta)$ on $\Omega_{0}$ should be even weaker or may even be reversed by taking into account the different evolution of the correlation function for different cosmological models.

For different contributions to $\bar{\Sigma}$ from different-sized dark halos, we vary the lower cutoff smoothing radius for the three models with $b_{8}^{-1} \Omega_{0}^{0.56}=0.57$. For $\Omega_{0}=1$, dark halos with smoothing radius $r_{0} \leq 10 \mathrm{Mpc}$ contribute over $90 \%$ of $\bar{\Sigma}$. For $\Omega_{0}=0.5$, dark halos with $r_{0} \leq 10 \mathrm{Mpc}$ account for about $75 \%$ of $\bar{\Sigma}$, and over $90 \%$ of the dark mass comes from dark halos with $r_{0} \leq 16 \mathrm{Mpc}$. For $\Omega_{0}=0.2$, dark halos with $r_{0} \leq 36 \mathrm{Mpc}$ contribute over $90 \%$ of $\bar{\Sigma}$. The differences shown here reflect different behaviors of power spectra of density fluctuations for the three models: the lower the $\Omega_{0}$ value, the greater the power on large scales.

Shown in Figure 4 is $\bar{\Sigma} / \Sigma_{\text {crit }}$ versus the cluster redshift, $z_{c}$, where $z_{s}=2, \theta=20^{\prime}$, and $b_{8}$ is determined by $b_{8}^{-1} \Omega_{0}^{0.56}=$ 0.57 . For all three models, $\Sigma / \Sigma_{\text {crit }}$ increases monotonically with $z_{c}$ for $\epsilon=-3$. With $\epsilon=-1.2$, the maximum of $\bar{\Sigma} / \Sigma_{\text {crit }}$ is at $z_{c} \sim 0.25, \sim 0.4$, and $\sim 0.6$ for $\Omega_{0}=1,0.5$, and 0.2 , respectively. With $\epsilon=0, \bar{\Sigma} / \Sigma_{\text {crit }}$ decreases monotonically for $\Omega_{0}=1$ and 0.5 , and has a mild peak at $z_{c} \sim 0.4$ for $\Omega_{0}=0.2$.

The amplification factor $\mu$ is plotted in Figure 5 for $\epsilon=-3,-1.2$, and 0 . The value of $b_{8}$ is determined by $b_{8}^{-1} \Omega_{0}^{0.56}=0.57, z_{c}=0.2$, and $z_{s}=2$. The velocity dispersion of clusters is taken to be $\sigma_{c}=1000 \mathrm{~km} \mathrm{~s}^{-1}$. For $\Omega_{0}=1$ and $\theta=20^{\prime}, \mu \approx 1.09,1.07$, and 1.06 for $\epsilon=-3,-1.2$, and 0 , respectively. For optically selected quasars, $\beta \approx 2.5$ (cf. $\beta$ definition in eq. [2.28]), and thus the respective enhancement factors $q$ are $1.14,1.11$, and 1.09. If we adopt $\beta \approx 3.5$

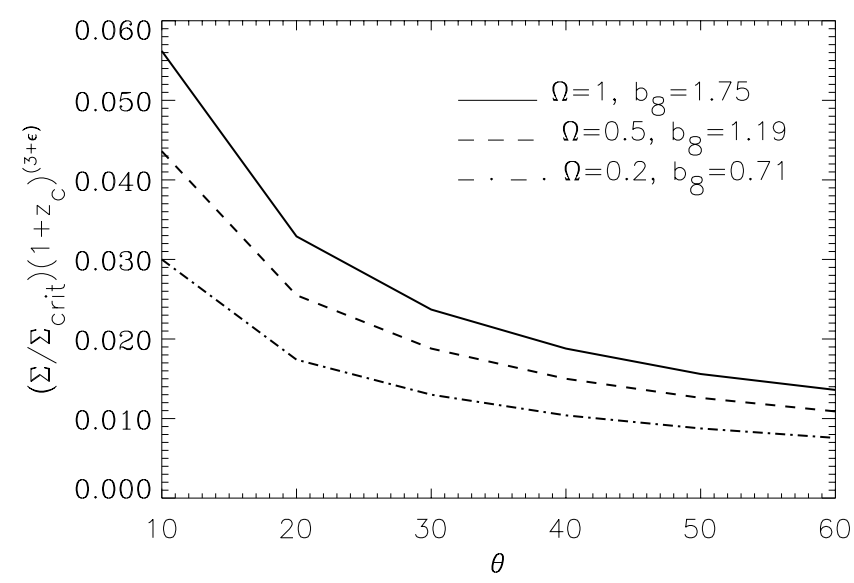

FIG. 3.-Plot of $\left(\bar{\Sigma} / \Sigma_{\text {crit }}\right)\left(1+z_{c}\right)^{(3+\epsilon)}$ vs. $\theta$ (in arcminutes), with bias factor $b_{8}=1.75,1.19$, and 0.71 for $\Omega_{0}=1,0.5$, and 0.2 , respectively, with $z_{s}=2$, and $z_{c}=0.2$. 


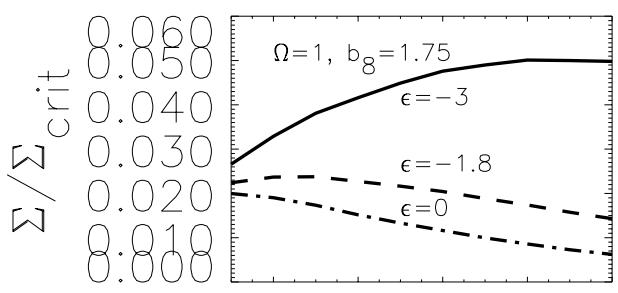

0.20 .40 .60 .81 .0
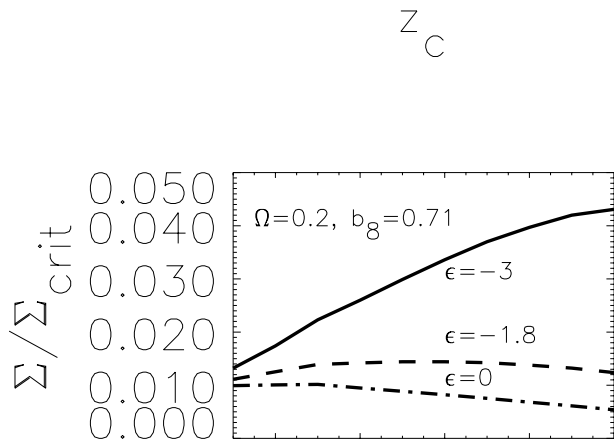

0.20 .40 .60 .81 .0

Z

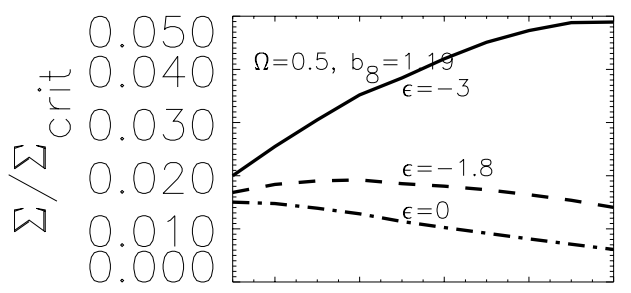

0.20 .40 .60 .81 .0

Z

FIG. 4. - Plot of $\bar{\Sigma} / \Sigma_{\text {crit }}$ vs. $z_{c}$, with source redshift $z_{s}=2$ and $\theta=20^{\prime}$

for optimal multiband-selected sources (Bartelmann \& Schneider 1992), the corresponding enhancement factors $q$ are 1.24, 1.18, and 1.16. For comparison, we show in Figure 6 the amplification factor $\mu$ with the same parameters as in

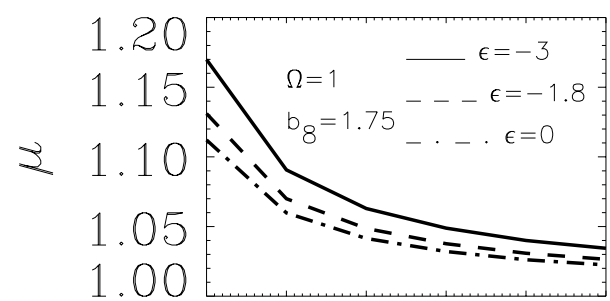

102030405060

$\theta$

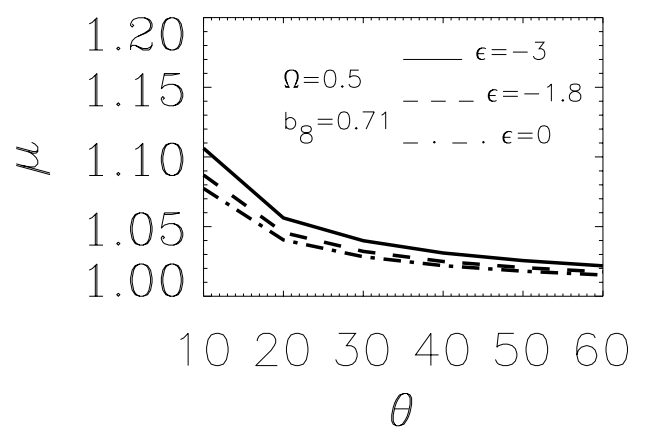

Figure 5, except that $b_{8}=1$. In this case, with $\Omega_{0}=1$ and $\theta=20^{\prime}, \mu \approx 1.16,1.12$, and 1.10 for $\epsilon=-3,-1.2$, and 0 , respectively. The corresponding $q$ 's are $1.25,1.19$, and 1.15 for $\beta \approx 2.5$, and $1.45,1.33$, and 1.27 for $\beta \approx 3.5$.

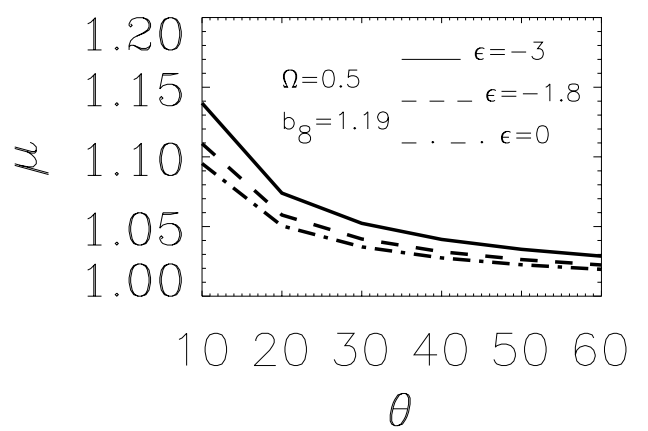

FIG. 5.-Lensing amplification factor $\mu$ vs. $\theta$ (in arcminutes), with source redshift $z_{s}=2$, cluster redshift $z_{c}=0.2$, and cluster velocity dispersion $\sigma_{v}=1000$ $\mathrm{km} \mathrm{s}^{-1}$. 

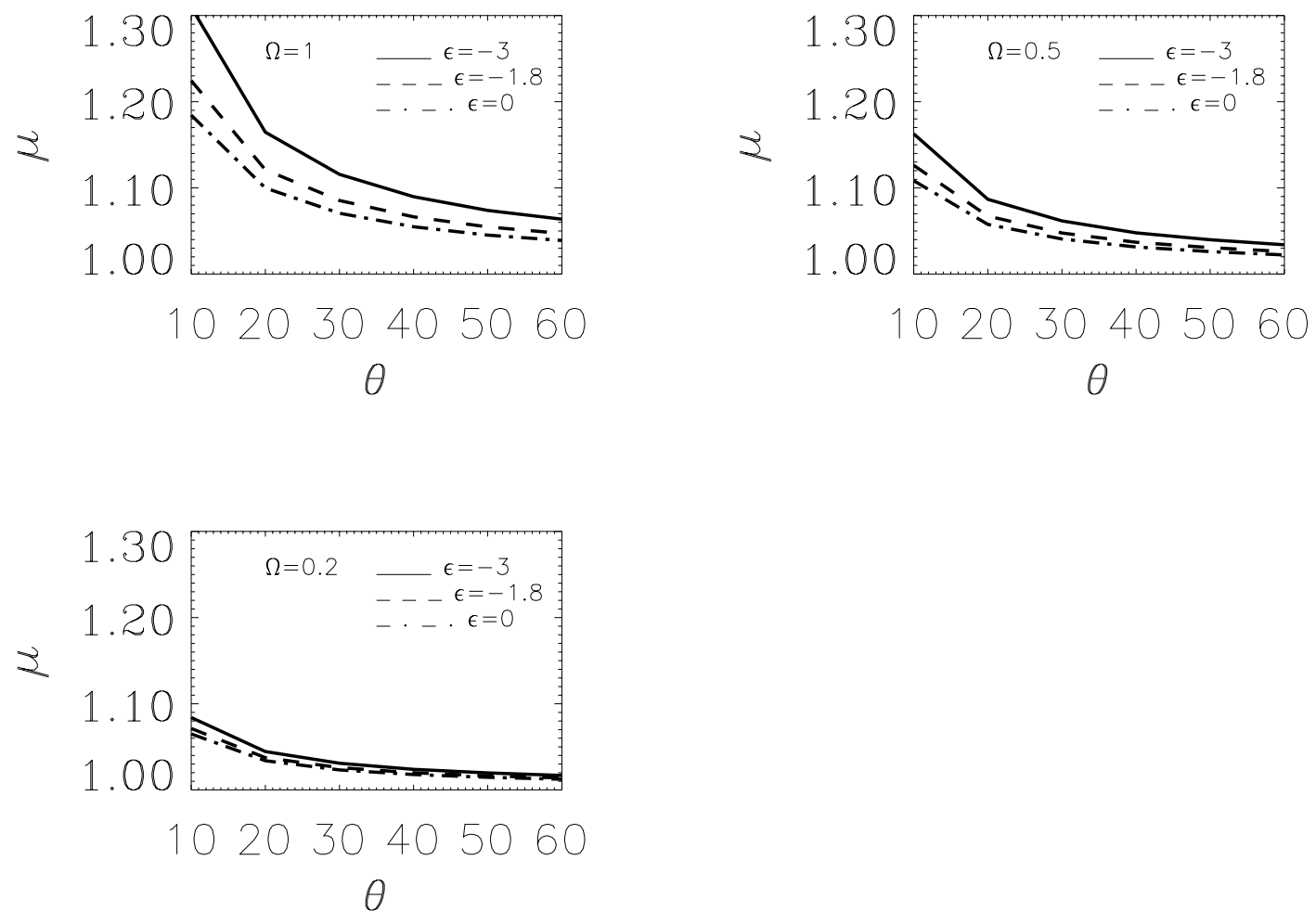

FIG. 6.-Lensing amplification factor $\mu$ vs. $\theta$ (in arcminutes), with source redshift $z_{s}=2$, cluster redshift $z_{c}=0.2$, and cluster velocity dispersion $\sigma_{v}=1000$ $\mathrm{km} \mathrm{s}^{-1}$, and a bias factor $b_{8}=1$ for all cases.

Observationally, the actually measured quantity is the average $q$ factor within $\theta_{0}$ around a cluster, namely,

$$
\bar{q}=\frac{\int_{\theta_{\min }}^{\theta_{0}} q(\theta) 2 \pi \theta d \theta}{\pi\left(\theta_{0}^{2}-\theta_{\min }^{2}\right)}
$$

where $\theta_{\min }$ is the minimum accessible angular distance to the cluster. From equations (2.26) and (2.29), $\bar{q}$ is approximately

$$
\bar{q} \approx\left(\frac{1}{1-2 \theta_{E} / \theta_{0}}\right)^{\beta-1}\left\{\frac{1}{\left[1-\left(\bar{\Sigma} / \Sigma_{\text {crit }}\right)\right]^{2}}\right\}^{\beta-1}
$$

where $\theta_{E}=4 \pi\left(\sigma_{c} / c\right)^{2}\left(D_{c s} / D_{s}\right)\left(1-\bar{\Sigma} / \Sigma_{\text {crit }}\right)^{-1}$. Equation (3.9) is valid for $\theta_{0} \gg \theta_{\min } \gg \theta_{E}$. For $\Omega_{0}=1, b_{8}=1.75, z_{s}=2$, $z_{c}=0.2, \epsilon=0, \sigma_{c}=1000 \mathrm{~km} \mathrm{~s}^{-1}$, and $\theta_{0}=20^{\prime}, \bar{q} \sim 1.12$ and $\sim 1.2$ for $\beta=2.5$ and 3.5, respectively. Our results for $\bar{q}$ are larger than estimates from the existing numerical simulations (Wu et al. 1997), but are still smaller than the observed enhancements.

Up to now, the environmental dark matter has been treated as being smoothly distributed in a disk. In reality, the distribution can be lumpy, which should increase the lensing amplification as lights closely passing by dark matter clumps. The effect of the compactness of the environmental dark matter distribution can be evaluated as follows (Schneider, Ehlers, \& Falco 1992). Let $p_{w}(\mu)$ be the average probability density of the amplification factor $\mu$ over a solid angle $w$. Then the average enhancement factor $\bar{q}_{d}$ due to the environmental dark matter is

$$
\bar{q}_{d}=\frac{1}{\Phi\left[A\left(z_{s}\right) S, z_{s}\right]\langle\mu\rangle_{w}} \int_{1}^{\infty} d \mu p_{w}(\mu) \Phi\left[\frac{A\left(z_{s}\right) S}{\mu}, z_{s}\right]
$$

where $\Phi\left(l, z_{s}\right)$ is the luminosity function of QSOs such that the comoving number density of QSOs at redshift $z_{s}$ with the luminosity greater than $L=l\left(c / H_{0}\right)^{2}$ is $\left(H_{0} / c\right)^{3} \Phi\left(l, z_{s}\right), S$ is the flux limit of a sample, the area $A(z)=4 \pi D^{2}(z)(1+z)^{4}$, and $\langle\mu\rangle_{w}$ is the average amplification factor over the solid angle $w$. For a uniform disk, $\langle\mu\rangle_{w}=\langle\mu\rangle=(1-\kappa)^{-2}$, with $\kappa \equiv \bar{\Sigma} / \Sigma_{\text {crit }}$, and $p_{w}(\mu)$ is modeled as

$$
p_{w}(\mu)= \begin{cases}\frac{q_{0}}{\mu^{3}} & \mu>\langle\mu\rangle, \\ p_{L} \delta\left(\mu-\mu_{L}\right) & \mu \leq\langle\mu\rangle,\end{cases}
$$

where

$$
\begin{gathered}
q_{0}=\frac{2 f_{c} \kappa}{(1-\kappa)^{2}}\left\{\left[1-\left(1-f_{c}\right) \kappa\right]^{2}+\left(f_{c} \kappa\right)^{2}\right\}^{-3 / 2}, \\
p_{L}=1-\frac{q_{0}}{2\langle\mu\rangle^{2}},
\end{gathered}
$$

and

$$
\mu_{L}=\frac{2\langle\mu\rangle\left(\langle\mu\rangle^{2}-q_{0}\right)}{2\langle\mu\rangle^{2}-q_{0}},
$$

where the compactness parameter, $f_{c}$, is the fractional dark matter mass that is in the form of compact clumps. For the luminosity function $\Phi$, one cannot use a single power-law distribution as in equation (2.28), because $\mu$ can be very large in the integral, such that $S / \mu$ can be lower than the break flux, $S_{B}$. Let us now consider an optically selected sample. The luminosity function $\Phi$ can be written as (cf. 
Schneider et al. 1992)

$$
\Phi\left[A\left(z_{s}\right) S, z_{s}\right]= \begin{cases}C\left(1-\beta / \alpha+\beta / \alpha s^{-\alpha}\right) & s<1, \\ C s^{-\beta} & s>1,\end{cases}
$$

where $C$ is a constant, $s \equiv S / S_{B}$, and $S_{B}$ is the flux corresponding to the break of the source counts, which occurs at the apparent magnitude of $m_{B} \approx 19.2$. The values of $\beta$ and $\alpha$ are chosen as $\beta=2.6$ and $\alpha=0.8$.

Then, for $s>1$, and $\langle\mu\rangle<s$, we have

$$
\begin{aligned}
\bar{q}_{d}= & \frac{p_{L} \mu_{L}^{\beta}}{\langle\mu\rangle}-\frac{q_{0}}{\beta-2}\langle\mu\rangle^{\beta-3} \\
& +\frac{q_{0}}{\langle\mu\rangle} s^{\beta-2}\left[\frac{1}{\beta-2}+\frac{1}{2}\left(1-\frac{\beta}{\alpha}\right)+\frac{\beta}{\alpha(2-\alpha)}\right] .
\end{aligned}
$$

For $s>1,\langle\mu\rangle>s$, and $\mu_{L}<s$,

$$
\begin{aligned}
\bar{q}_{d}= & \frac{p_{L} \mu_{L}^{\beta}}{\langle\mu\rangle}+\frac{q_{0}}{\langle\mu\rangle} s^{\beta-2} \\
& \times\left[\frac{\beta}{\alpha(2-\alpha)}\left(\frac{\langle\mu\rangle}{s}\right)^{\alpha-2}+\frac{1}{2}\left(1-\frac{\beta}{\alpha}\right)\left(\frac{\langle\mu\rangle}{s}\right)^{-2}\right] .
\end{aligned}
$$

For $s>1,\langle\mu\rangle>s$, and $\mu_{L}>s$,

$$
\begin{aligned}
\bar{q}_{d}= & \frac{p_{L}}{\langle\mu\rangle} \frac{1-\beta / \alpha+(\beta / \alpha) s^{-\alpha} \mu_{L}^{\alpha}}{s^{-\beta}} \\
& +\frac{q_{0}}{\langle\mu\rangle} s^{\beta-2}\left[\frac{\beta}{\alpha(2-\alpha)}\left(\frac{\langle\mu\rangle}{s}\right)^{\alpha-2}\right. \\
& \left.+\frac{1}{2}\left(1-\frac{\beta}{\alpha}\right)\left(\frac{\langle\mu\rangle}{s}\right)^{-2}\right] .
\end{aligned}
$$

In the regime of weak lensing with $\bar{q}_{d}-1 \ll 1$ and $\bar{q}_{c}$ $-1 \ll 1$, where $\bar{q}_{c}$ is the average enhancement factor due to the central cluster, the total average enhancement factor within $\theta$ can be approximated by

$$
\begin{aligned}
\bar{q} & =1+\left(\bar{q}_{c}-1\right)+\left(\bar{q}_{d}-1\right) \\
& =(\beta-1) \frac{8 \pi\left(\sigma_{c} / c\right)^{2}\left(D_{c s} / D_{s}\right)}{\theta}+\bar{q}_{d} .
\end{aligned}
$$

We show $\bar{q}$ versus $\theta$ in arcminutes in Figure 7. The model parameters are $\Omega_{0}=1, b_{8}=1.75, \epsilon=0, z_{s}=2, z_{c}=0.2$, and $\sigma_{c}=1000 \mathrm{~km} \mathrm{~s}^{-1}$. The solid lines show the results from equation (3.16), including the compact component with $f_{c}=1$, and the dashed line shows the results from equation (3.9), without considering the lumpy structures (i.e., $f_{c}=0$ ). The three solid lines correspond to different luminosity limits. From top to bottom, the apparent magnitude difference $\Delta m \equiv m_{L}-m_{B}=-1.0,-0.7$, and -0.5 , respectively, where $\Delta m$ is related to $s$ through $s=10^{-0.4 \Delta m}$. We see that $\bar{q}\left(f_{c}=1\right)>\bar{q}\left(f_{c}=0\right)$. For any other $f_{c}$ values $0<f_{c}<1, \bar{q}$ falls between $\bar{q}\left(f_{c}=1\right)$ and $\bar{q}\left(f_{c}=0\right)$. For the QSO catalog used by Rodrigues-Williams \& Hogan (1994), $\Delta m=0.7$. The observed $\bar{q}$ on the angular scale of $\sim 50^{\prime}$ is $\sim 1.7$, which is considerably larger than our theoretical result. In fact, independent of specific lensing models, the maximum $\bar{q}$ from gravitational lensing can be estimated theoretically by using the optimal $p_{w}(\mu)$ in equation (3.10) (cf. Schneider et al. 1992). For the small $\bar{\Sigma}$ derived from dark matter-cluster correlations, $\bar{q}_{\max }$ is again much smaller than the observed value at angular scales around $50^{\prime}$.

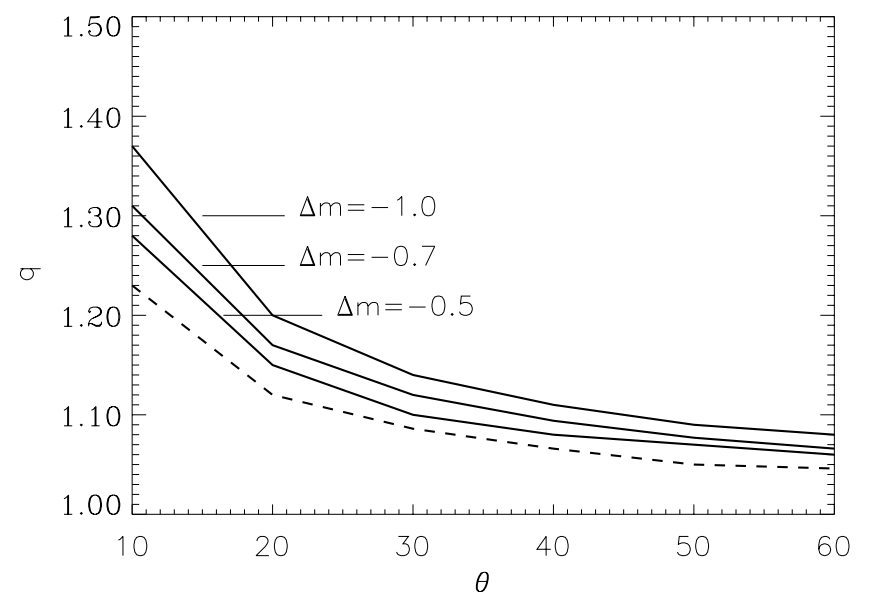

FIG. 7.-Average enhancement factor $\bar{q}$ vs. $\theta$ (in arcminutes). The parameters are $\Omega_{0}=1, b_{8}=1.75, \epsilon=0, z_{s}=2, z_{c}=0.2$, and $\sigma_{c}=1000 \mathrm{~km}$ $\mathrm{s}^{-1}$. The solid lines represent the compactness parameter $f_{c}=1$, with respective apparent magnitude difference $\Delta m=-1.0,-0.7$, and -0.5 (top to bottom). The dashed line shows the result from the smoothed dark matter distribution $\left(f_{c}=0\right)$.

We now turn to the cluster mass estimate from weaklensing effects. The environmental dark matter, $\bar{\Sigma}(\theta)$, contributes to weak distortions of background galaxies, and thus affects the cluster masses determined by weak lensing (Kaiser \& Squires 1993). From the inversion of weak distortions, the projected mass within radius $\eta_{0}$ is given by (Fahlman et al. 1994)

$$
M\left(\eta_{0}\right)=\pi \eta_{0}^{2}\left[\bar{\sigma}\left(\eta<\eta_{0}\right)-\bar{\sigma}\left(\eta_{0}<\eta<\eta_{\max }\right)\right],
$$

where $\bar{\sigma}\left(\eta<\eta_{0}\right)$ is the total average surface mass density within $\eta_{0}$, and $\bar{\sigma}\left(\eta_{0}<\eta<\eta_{\max }\right)$ is the total average surface mass density within the annulus $\eta_{0}<\eta<\eta_{\max }$. Let $\bar{\Sigma}_{c}$ be the average surface mass density of the real cluster; then

$$
\bar{\sigma}\left(\eta<\eta_{0}\right)=\bar{\Sigma}_{c}\left(\eta<\eta_{0}\right)+\bar{\Sigma}\left(\eta<\eta_{0}\right),
$$

and

$$
\bar{\sigma}\left(\eta_{0}<\eta<\eta_{\max }\right)=\bar{\Sigma}_{c}\left(\eta_{0}<\eta<\eta_{\max }\right)+\bar{\Sigma}\left(\eta_{0}<\eta<\eta_{\max }\right) .
$$

We use the singular isothermal sphere to describe the cluster density profile; then

$$
\bar{\Sigma}_{c}\left(\eta<\eta_{0}\right)=\frac{\sigma_{c}^{2}}{G} \frac{1}{\eta_{0}}
$$

and

$$
\begin{aligned}
\bar{\Sigma}_{c}\left(\eta_{0}<\eta<\eta_{\max }\right) & =\frac{\bar{\Sigma}_{c}\left(\eta<\eta_{\max }\right) \pi \eta_{\max }^{2}-\bar{\Sigma}_{c}\left(\eta<\eta_{0}\right) \pi \eta_{0}^{2}}{\pi\left(\eta_{\max }^{2}-\eta_{0}^{2}\right)} \\
& =\frac{\sigma_{c}^{2} / G}{\eta_{0}+\eta_{\max }}
\end{aligned}
$$

Therefore,

$$
\begin{aligned}
M\left(\eta_{0}\right)= & \frac{\sigma_{c}^{2}}{G} \frac{1}{\eta_{0}} \pi \eta_{0}^{2}+\bar{\Sigma}\left(\eta<\eta_{0}\right) \pi \eta_{0}^{2}-\frac{\sigma_{c}^{2} / G}{\eta_{0}+\eta_{\max }} \pi \eta_{0}^{2} \\
& -\frac{\bar{\Sigma}\left(\eta<\eta_{\max }\right) \pi \eta_{\max }^{2}-\bar{\Sigma}\left(\eta<\eta_{0}\right) \pi \eta_{0}^{2}}{\pi\left(\eta_{\max }^{2}-\eta_{0}^{2}\right)} \pi \eta_{0}^{2} .
\end{aligned}
$$

If $M_{c}$ stands for the true mass of the cluster, then the weak- 


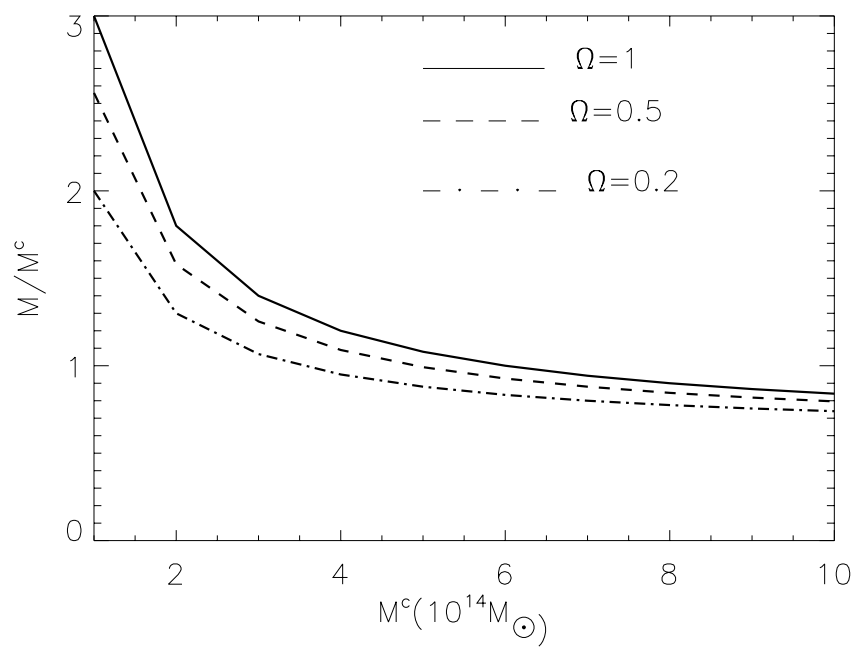

FIG. 8. - Plot of $M / M_{c}$ vs. $M_{c}$, with cluster redshift $z_{c}=0.3, \eta_{0}=1.0$ Mpc, $\eta_{\max }=1.5 \mathrm{Mpc}$, and bias factor $b_{8}=1.75,1.19$, and 0.71 for $\Omega_{0}=1$, 0.5 , and 0.2 , respectively.

lensing-estimated mass, $M$, is

$$
\begin{aligned}
M\left(\eta_{0}\right)=M_{c}+\left[\frac{\bar{\Sigma}\left(\eta<\eta_{0}\right)-\bar{\Sigma}\left(\eta<\eta_{\max }\right)}{1-\left(\eta_{0} / \eta_{\max }\right)^{2}} \pi \eta_{0}^{2}\right. & \\
& \left.-\frac{M_{c}}{1+\left(\eta_{\max } / \eta_{0}\right)}\right] .
\end{aligned}
$$

We see that $M>M_{c}$ for relatively small $M_{c}$, and $M<M_{c}$ for massive clusters.

Shown in Figure 8 is a plot of $M / M_{c}$ versus $M_{c}$. The parameters are $z_{c}=0.3, \eta_{0}=1.0 \mathrm{Mpc}, \eta_{\max }=1.5 \mathrm{Mpc}$, and $b_{8}=1.75,1.19$, and 0.71 for $\Omega_{0}=1,0.5$, and 0.2 , respectively. We have used $\epsilon=0$ for all three models. We derive $\bar{\Sigma}$ at $\eta_{0}$ and $\eta_{\max }$ by extrapolating $\bar{\Sigma}$ from $\theta=20^{\prime}$ (which, at $z_{c}=0.3$, corresponds to $\eta=6.6,6.8$, and $7.0 \mathrm{Mpc}$ for $\Omega_{0}=$ $1,0.5$, and 0.2 , respectively) based on the approximate relation $\bar{\Sigma} \propto \eta^{-0.8}$. We found that $M / M_{c}>1$ for $M_{c}<6.0,4.9$, and $3.5 \times 10^{14} M_{\odot}$ for $\Omega_{0}=1,0.5$, and 0.2 , respectively. Our results qualitatively agree with the simulation results of Relinsky \& Bartelmann (1999). An important point of these results is that the cluster mass determined from weaklensing analyses is not always a lower bound on the real mass of a cluster, and the estimated mass could be as high as twice the true cluster mass. For a fixed $\eta_{0}$, the larger the ratio $\eta_{\max } / \eta_{0}$, the larger the $M_{c}$ in order to have $M / M_{c}<1$.

\section{DISCUSSION}

We have studied the environmental dark matter contribution to the projected surface mass density around clusters due to the cluster-dark halo correlation. At a typical cluster redshift $z_{c}=0.2$, the surface mass density of the environmental dark matter can be comparable to the surface mass density of the cluster itself. The total average enhancement factor, $\bar{q}$, for background QSOs can reach $\sim 1.2$ for $\beta=3.5$, and $\bar{q} \sim 1.12$ for $\beta=2.5$ at $\theta=20^{\prime}$ for $\Omega_{0}=1, \epsilon=0$, and $b_{8}=1.75$, as compared to $\bar{q} \sim 1.1$ and $\bar{q} \sim 1.06$ caused solely by the cluster itself for the respective $\beta$ values. Our results are higher than those of simulations (Wu et al. 1997). The limited resolution of numerical simulations may be one reason for this discrepancy. On the other hand, in our analyses we have assumed that the power-law dark halocluster correlation function is valid up to very small separations. This assumption may not be adequate and can result in an overestimate of the environmental dark matter contribution. In addition, strictly speaking, the linear bias in the form of equations (3.6) and (3.7) is valid only in the high peak limit, with $\delta_{c} / \Delta \gg 1$ (cf. Bardeen et al. 1986). For the galaxy bias, equation (3.7) is a reasonable approximation (cf. Bardeen et al. 1986). For relatively small dark halos, however, $\delta_{c} / \Delta$ can be less than 1 . Mo \& White (1996) derived an analytical formula for the linear bias of dark halos from the Press-Schechter theory. For dark halos formed at redshift $z$, the corresponding linear bias, also measured at $z$, is given by

$$
\tilde{b}_{d}\left(r_{0}, z\right)=\frac{\delta_{c}^{2}(0) / \Delta^{2}\left(r_{0}, z\right)-1}{\delta_{c}(0)}+1
$$

Note that for the same set of halos, the bias would be different when measured at different redshifts (Mo \& White 1996). For halos with $\Delta\left(r_{0}, z\right)>\delta_{c}(0)$, it follows that $\tilde{b}_{d}\left(r_{0}, z\right)<1$, i.e., those halos are antibiased with respect to the dark matter distribution. In comparison, the bias $b_{d}\left(r_{0}, z\right)$, as given by equation (3.6), always remains larger than 1. Numerical simulations show that equation (4.1) describes well the linear bias of dark halos with $\delta_{c}(0) / \Delta\left(r_{0}, z\right)>1$ (note that in this limit, $b_{d} \approx \tilde{b}_{d}$ ). For small halos, it is found that they are indeed antibiased relative to the distribution of dark matter, in qualitative agreement with equation (4.1). Quantitatively, equation (4.1) tends to overestimate the antibias, in the sense that the bias from numerical simulations is larger than that predicted by equation (4.1) (Jing 1998, 1999; Catelan, Matarrese, \& Porciani 1998). An inclusion of the possible antibias for small dark halos will decrease the QSO-cluster correlation in our analyses. One should also note that the linear scale corresponding to $\theta=10^{\prime}$ is about $2.6 \mathrm{Mpc}$ at $z_{c}=0.2$, and thus the nonlinear bias may be important at relatively small angular scales. On the other hand, it is not expected that our results will be significantly changed by including the nonlinear biasing effect. Nonetheless, with $\Omega_{0}=1$ and $b_{8} \sim$ 2 , our results do show, consistent with the conclusion of $\mathrm{Wu}$ et al. (1997), that the theoretical enhancement $q$ factor remains smaller than the observed value. This holds true for low- $\Omega$ open universes. For a low $\Omega$ flat universe with a nonzero cosmological constant, the $q$ factor at $z_{c} \sim 0.2$ is different from that of the low- $\Omega$ open universe with the same $\Omega_{0}$, mainly because of the geometric differences in the two universes, and therefore the different critical surface mass density, $\Sigma_{\text {crit }}$. We do not expect that $q$ would be significantly higher in a low- $\Omega$ flat universe than that in a low- $\Omega$ open universe. Thus, the observed large $q$ poses a challenge. We note that our calculations are based on statistically averaged dark halo-cluster correlations. Observationally selected clusters possibly only represent a sample of clusters biased toward large masses for various observational reasons (e.g., Rodrigues-Williams \& Hogan 1994), and thus the observed large $q$ could be a biased result for massive clusters. If we take $\sigma_{c}=2000 \mathrm{~km} \mathrm{~s}^{-1}$ and assume that the dark halo-cluster correlation is twice the value used in our calculations, then $\bar{q} \sim 1.46$ and $\sim 1.2$ at $\theta=20^{\prime}$ and $\theta=50^{\prime}$, respectively, for $\beta=2.5, \Omega_{0}=1, b_{8}=1.75, \epsilon=0$, and $z_{c}=$ 0.2 , which is still less than the observed value $(\bar{q} \sim 1.7$ at $\theta_{0} \sim 50^{\prime}$; cf. Rodrigues-Williams \& Hogan 1994). This ad 
hoc example indicates that the biasing effect toward massive clusters still may not fully explain the observational results. The existence of lumpy structures in the distribution of the environmental dark matter tends to increase the $\bar{q}$ value, but these increases are not yet sufficient to account for the observed strong QSO-cluster correlations. On the other hand, because of the small number of observed QSOs, it is difficult to guage their background surface number density properly, and thus the observed high $q$ factor may not be as significant as it appears (e.g., Seitz \& Schneider 1995). There are other observational issues to be further explored (e.g., Wu et al. 1997).

We have shown in this paper in a very clear manner (cf. eq. [3.18]) that theoretically, the cluster mass estimated from the weak-lensing effect is not always a lower bound to the real cluster mass. For relatively small clusters, the estimated mass is higher than the real one. Even though the real cluster density profile and the distribution of its surrounding dark matter are much more complex than a singular isothermal sphere and a uniform sheet can represent, the relation of $M / M_{c}$ and $M_{c}$ from our simplified analyses agrees qualitatively with the simulation results of Reblinsky
\& Bartelmann (1999). Since distortions of background galaxies are easily detected around very massive clusters, the cluster mass determined observationally could indeed be an underestimate. However, if the observed large enhancement of QSOs around foreground clusters is true and is caused by the gravitational lensing of large-scale structures not yet fully understood, the same large-scale structures would have a severe effect on the cluster mass determination from weak-lensing analyses. In principle, the cluster mass inferred from the strong-lensing effect should also be affected by the environmental dark matter, particularly because there is no subtraction involved in the mass estimate procedure. However, the strong lensing can only occur at very central regions of very heavy clusters, and we therefore expect negligible strong lensing effects from the environmental dark matter as compared to those caused by massive clusters themselves.

We thank the referee for the constructive comments. This research was supported in part by grants NSC89-2112M002-037 from the National Science Council, Republic of China.
Bardeen, J. M., Bond, J. R., Kaiser, N., \& Szalay, A. S. 1986, ApJ, 304, 15 Bartelmann, M. 1995, A\&A, 298, 661

Bartelmann, M., \& Schneider, P. 1992, A\&A, 259, 413 . 1993a, A\&A, 268, 1 1993b, A\&A, 271, 421 1999, A\&A, 345, 17

Bartsch, A., Schneider, P., \& Bartelmann, M. 1997, A\&A, 319, 375

Benitez, N., Martinez-Gonzalez, E., \& Martin-Mirones, J. M. 1997, A\&A, 321, L1

Catelan, P., Matarrese, S., \& Porciani, C. 1998, ApJ, 502, L1

Cen, R. 1997, ApJ, 485, 39

Cen, R., Gott, J. R., III, Ostriker, J. P., \& Turner, E. L. 1994, ApJ, 423, 1

Dolag, B., \& Bartelmann, M. 1997, MNRAS, 291, 446

Efstathiou, G., Bond, J. R., \& White, S. D. M. 1992, MNRAS, 258, L1

Fahlman, G., Kaiser, N., Squires, G., \& Woods, D. 1994, ApJ, 437, 56

Fort, B., Mellier, Y., Dantel-Fort, M., Bonnet, H., \& Kneib, J.-P. 1996, A\&A, 310, 705

Fugmann, W. 1990, A\&A, 240, 11

Hawkins, M. R. S. 1997, A\&A, 328, L25

Jain, B., \& Seljak, U. 1997, ApJ, 484, 560

Jaroszynski, M., Park, C., Paczynski, B., \& Gott, J. R. 1990, ApJ, 365, 22

Jing, Y. P. 1998, ApJ, 503, L9 1999 , ApJ, 515, L45

Kaiser, N. 1998, ApJ, 498, 26

Kaiser, N., \& Squires, G. 1993, ApJ, 404, 441

Kochanek, C. S. 1995, ApJ, 453, 545

Koo, D. C., \& Szalay, A. S. 1984, ApJ, 282, 390

\section{REFERENCES}

Mo, H. J., \& White, S. D. M. 1996, MNRAS, 282, 347

Narayan, R. 1989, ApJ, 339, L53

Narayan, R., \& White, S. D. M. 1988, MNRAS, 231, P97

Peacock, J. A., \& Dodds, S. J. 1994, MNRAS, 267, 1020

Peebles, P. J. E. 1980, The Large-Scale Structure of the Universe (Princeton: Princeton Univ. Press)

Press, W., \& Schechter, P. 1974, ApJ, 187, 425

Reblinsky, K., \& Bartelmann, M. 1999, A\&A, 345, 1

Rodrigues-Williams, L. L., \& Hawkins, M. R. S. 1995, in AIP Conf. Proc.

336, Dark Matter, ed. S. S. Holt, \& C. L. Bennett (New York: AIP), 310

Rodrigues-Williams, L. L., \& Hogan, C. J. 1994, AJ, 107, 451

Sanz, J. L., Martinez-Gonzalez, E., \& Benitez, N. 1997, MNRAS, 291, 418

Schneider, P. 1989, A\&A, 221, 221

Schneider, P., Ehlers, J., \& Falco, E. E. 1992, Gravitational Lenses (Berlin: Springer)

Schneider, P., van Waerbeke, L., Mellier, Y., Jain, B., Seitz, S., \& Fort, B. 1998, A\&A, 333, 767

Seitz, S., \& Schneider, P. 1995, A\&A, 302, 9

Shepherd, C. W., Carlberg, R. G., Yee, H. K. C., \& Ellingson, E. 1997, ApJ, 479,82

Wambsganss, J., Cen, R., \& Ostriker, J. P. 1998, ApJ, 494, 29

White, S. D. M., Efstathiou, G., \& Frenk, C. S. 1993, MNRAS, 262, 1023

Wu, X. P., \& Fang, L. Z. 1996, ApJ, 461, L5

Wu, X. P., Fang, L. Z., Zhu, Z. H., \& Qin, B. 1996, ApJ, 471, 575

Wu, X. P., \& Han, J. L. 1995, MNRAS, 272, 705

Wu, X. P., Zhu, X. H., Jing, Y. P., \& Fang, L. Z. 1997, ApJ, 488, 557 